\title{
EXPERIMENTAL OBSERVATION OF THE TEMPERATURE AT THE LIQUID/GAS INTERFACE
}

\author{
$V$. D. Velicanov ${ }^{1}, E$. G. Orlova ${ }^{1, *}, A$. S. Saigash ${ }^{1}$, and $O$. S. Korneva ${ }^{1}$ \\ ${ }^{1}$ National Research Tomsk Polytechnic University, 634050 Tomsk, Russia
}

\begin{abstract}
Experimental studies on measurements of the temperature profile in the vicinity of the evaporating distilled water layer were carried out. The temperature was measured with a chromel-alumel thermocouple, when it moves under the action of the precision actuator directed perpendicular to the evaporating layer. According to the experimental results the fluctuation in temperature of the air and liquid was found in the immediate vicinity of the liquid/gas interface. However, temperature jumps near the interface was not observed.
\end{abstract}

\section{Introduction}

The main problem at modelling of processes involving the phase transitions is the determination of the boundary conditions prescribed on the thermodynamic variables, such as temperature and partial pressure on the liquid/gas interface. The computation of heat transfer at condensation or evaporation of liquid films is the example of these processes [17]. There is a nonequilibrium region in the vicinity of the interface due to the small mean free path of vapour molecules. It means that the temperature and vapour pressure are deflected from the surface temperature and the saturation pressure at this temperature [8]. That is, there is a possibility of an abrupt change of temperature and pressure [9] in the vicinity of the liquid/gas interface, even for Knudsen numbers close to zero. To date, the vapours generated above the liquid surface are considered as an ideal gas in the modelling of the evaporation or the condensation. In this case, the temperature and pressure gradients at the interface are determined by solving the problem on definition of the boundary conditions using Boltzmann equation.

The mathematical model of the liquid evaporation including the temperature gradient at the interface was developed in [9]. It was found that the evaporation rate of liquid is higher by three orders, if the temperature and jumps boundary conditions were used in accordance with the kinetic theory in comparison with using the "continuity" of the temperature and pressure [9].

The temperature field at the liquid/gas interface was studied experimentally during evaporation of the ultrapure water obtained by of Milli-Q purification system [10]. The constant thickness of the layer was kept by a dispenser device (syringe pump). The

* Corresponding author: $\underline{\mathrm{fdv} @ \text { tpu.ru }}$ 
temperature was recorded by the thermocouples with a diameter of $150 \mu \mathrm{m}$, located on the movable part of the linear actuator. The thickness of the liquid layer and the surface temperature were kept constant and were equal to $1.75 \mathrm{~mm}$ and $31{ }^{\circ} \mathrm{C}, 52{ }^{\circ} \mathrm{C}, 92{ }^{\circ} \mathrm{C}$, respectively. According to the results of the experiments the temperature gradient at the interface was recorded. The average value of the gradient was $1.3^{\circ} \mathrm{C}$, and it is increased with increasing substrate temperature (the heater). For example, the gradients for temperatures of 31,52 and $92{ }^{\circ} \mathrm{C}$ were $0.2,1$ and $3{ }^{\circ} \mathrm{C}$, respectively.

The temperature gradient indicates the presence of thermal resistance on the liquid surface. If this resistance is present, it is necessary to consider the inequality of evaporation and condensation coefficients in the numerical study of these processes.

The aim of this work is to determine experimentally the existence of the temperature gradient at the liquid/gas interface under the condition of thin liquid layer evaporation into the atmosphere.

\section{Experimental procedures}

The studies were carried out on the experimental setup (fig. 1) with using the equipment of the shadow method $[11,12]$.

The temperatures of the upper and lower surfaces of the heater, liquid and environment were measured using six chromel-alumel thermocouples 6 (OMEGA 5TC-TT-K-40-36). Two by two thermocouples were located on the top and bottom surfaces of the metal plate, one in the liquid layer and one in the environment inside the box.

Signals from thermocouples, were transferred to the controller-ADC 7 (NI cDAQ-9171) further to the personal computer 12, where the processing and displaying on the screen of data were implemented in software developed in LABVIEW Signal Express 2012. The substrate used was PTFE; the liquid was distilled water. The surface temperature of the substrate was kept constant equal to $56^{\circ} \mathrm{C}$.

Temperature measurements in the immediate vicinity of the liquid/gas interface were performed using the calibrated thermocouple K type (OMEGA 5TC-TT-K-40-36) with a thermojunction of 80 microns. The responses were tested with standard resistance thermometers ETS-100 with the error in the test temperature ranges of $0.05{ }^{\circ} \mathrm{C}$. The estimation of measurement errors was carried out as described in [13]. According to results of calculation the error does not exceed $2 \%$. 


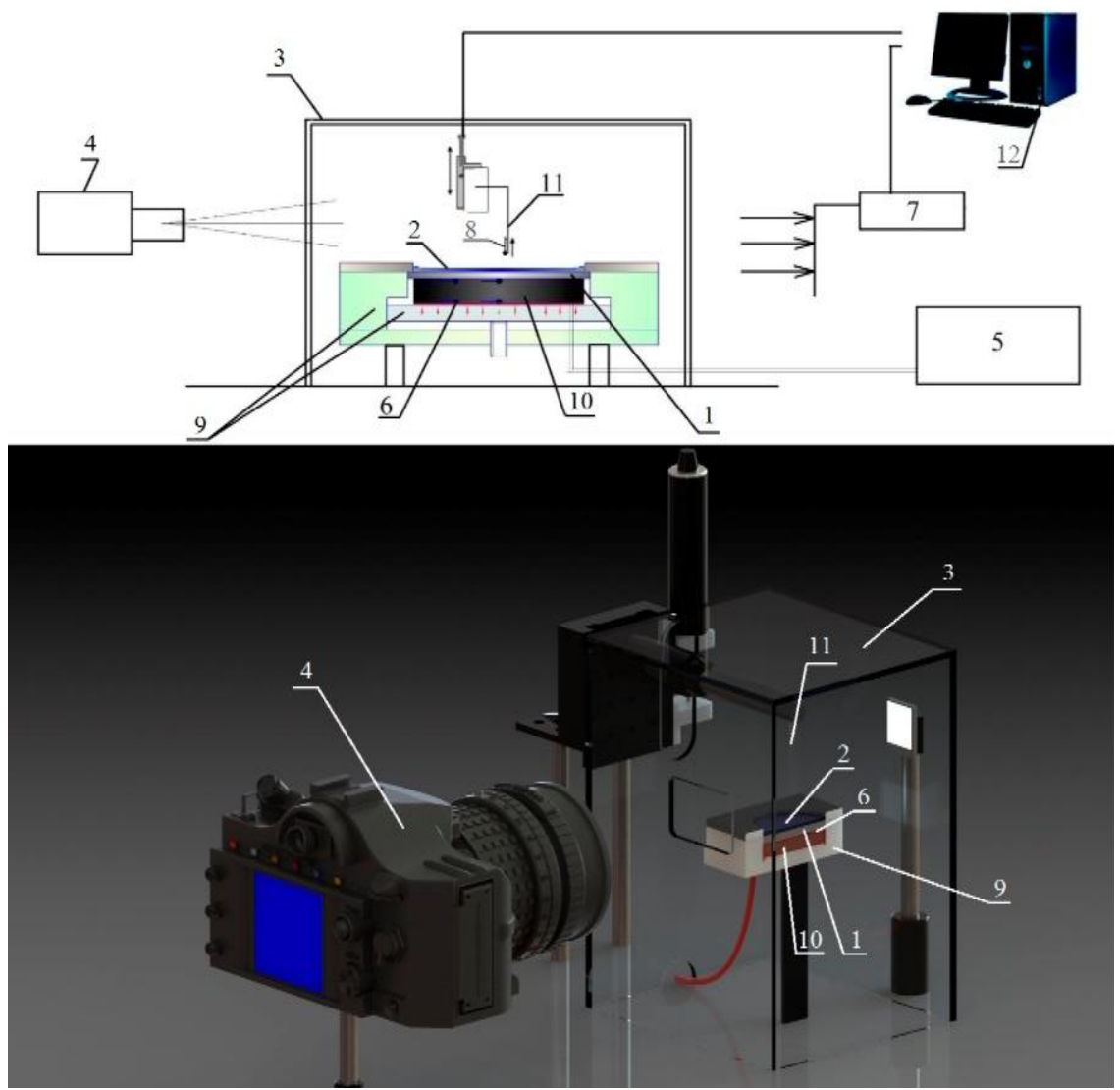

Fig. 1. Scheme of the experimental setup: 1 - substrate; 2 - liquid; 3 - transparent box; 4 photographic camera; 5 - power supply; 6, 8 - thermocouples; 7 - analog-to-digital converter; 9 thermal insulating wall; 10 - metal plate; 11 - gauge (needle of the actuator); 12 - personal computer.

The position of the micro-thermocouple was controlled by the precision actuator Zaber with increment of $1 \mathrm{~mm}$. The travel speed of the movable part of the actuator was 100 $\mu \mathrm{m} / \mathrm{s}$.

Distilled water was dosed to the substrate after heating its surface up to a predetermined temperature of $56^{\circ} \mathrm{C}$. In this case the layer of air at a distance of $2 \mathrm{~mm}$ above the surface was heated to $55^{\circ} \mathrm{C}$ (fig. 2). Next, the actuator was turned on and the thermocouple moved to the surface of liquid/gas interface.

\section{Results and discussion}

Figure 2 presents the typical experimental dependence of the temperature change from the position of thermocouple in the vertical plane relative to the liquid/gas interface.

The point 0.0 on the vertical axis corresponds to the liquid/gas interface. Negative values of this axis are the coordinates of the liquid phase (distilled water); the positive ones are the gas phase (air). Presented dependence (fig. 2) was plotted based on the results of five experiments as the arithmetic mean of the recorded data.

The fluctuation in temperature of the air and liquid in the immediate vicinity of the liquid/gas interface was found according to analyses of the dependence (fig. 2). What is more, no temperature jumps were observed on a distance of \pm 80 microns from the interface 
(fig. 2). This distance corresponds to a thermojunction of a thermocouple. The fluctuation nature of the temperature can be explained as follows. When liquid is dozed on the surface the under layer, which contacts to the surface, warms up. The temperature gradient and the density gradient appear; they directed from the interface. It leads to liquid convection. The top colder layer of liquid moves down, and warmed layer rises to the interface. It is obvious that the circulation of liquid affects the movement of the air near the interface. And as a result, the fluctuations of the temperature appear. Under the normal conditions with atmospheric pressure this thickness is equal to $1 \mathrm{~mm}$ for water vapour [9].

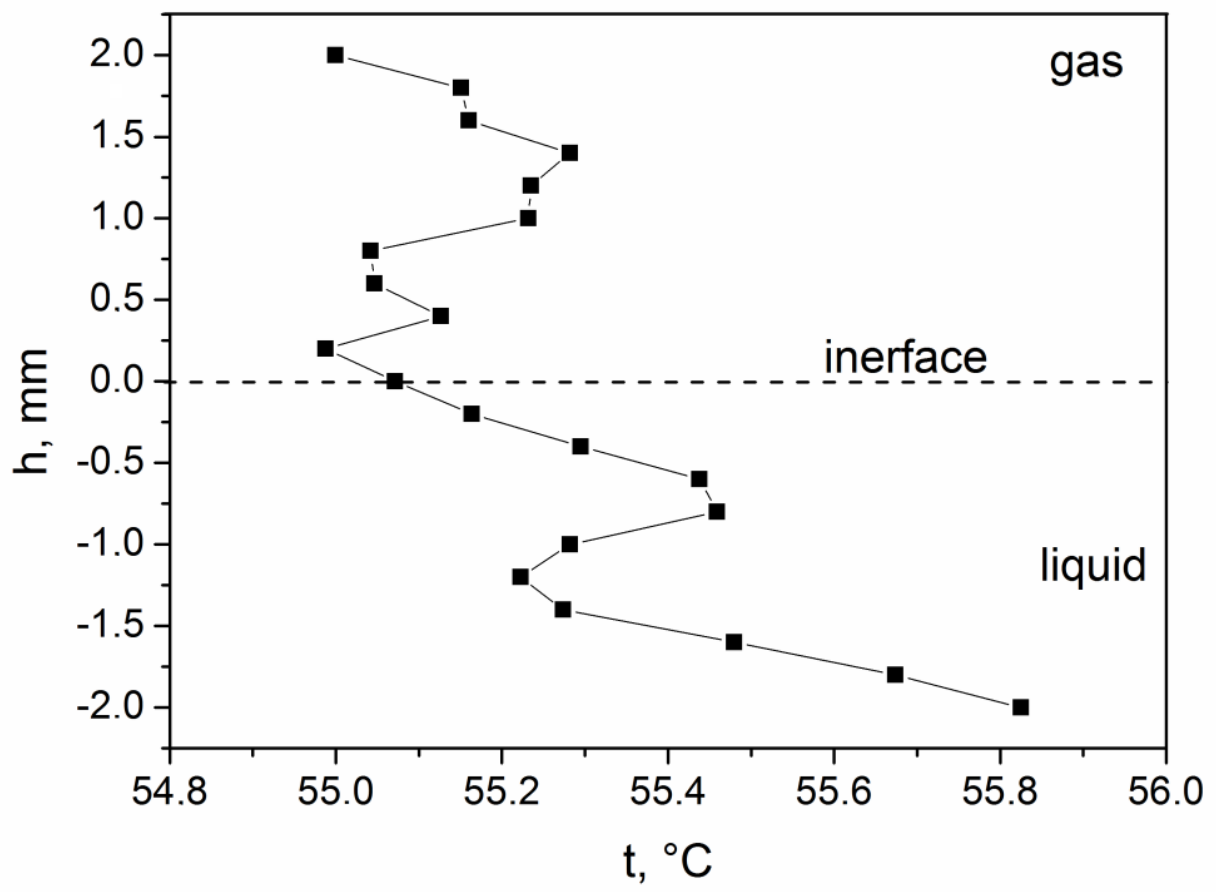

Fig. 2. Dependence of the temperature from the position of thermocouple in the vertical plane relative to the liquid/gas interface.

Therefore, for further solving the problem of determining the thermodynamic parameters at the interface and their influence on the process of evaporation and condensation it is necessary to conduct the experimental studies using thermocouple with a diameter of a thermojunction up to $10 \mu \mathrm{m}$.

According to results of the experiments the condensate layer was found to form on the thermocouple during its movement to the interface. To the best of our knowledge, it will affect the recorded parameters.

So, it can be concluded that it is necessary to use a thermocouple with horizontally ("telescoping") arranged electrodes. Such arrangement will correspond to the isotherms of the liquid/gas interface and will reduce the heat loss associated with the temperature gradient across the electrodes of thermocouples. Also it will reduce the thickness of the condensate layer forming on the surface of the thermocouple.

\section{Acknowledgments}

The reported study was supported by the grant of President of Russian Federation for the government support of young Russian scientists (MK-6810.2016.8). 


\section{References}

1. S. Ya Misyura, Chemical Physics Letters 583, 34 (2013)

2. V.P. Lebedev, V. V. Lemanov, S. Ya. Misyura, V. I. Terekhov, Fluid Dynamics 28, 624 (1993)

3. K. Batischeva, E. Orlova, D. Feoktistov, MATEC Web Conf. 19, 01001 (2014)

4. I. Afanasyev, E. Orlova, D. Feoktistov, EPJ Web Conf. 82, 01054 (2015)

5. D.O. Glushkov, G.V. Kuznetsov, P.A. Strizhak, Thermal Science 19, 1541 (2015)

6. A.S. Krasnoshlykov, V.I. Maksimov, EPJ Web Conf. 82, 01046 (2015)

7. V.I. Maksimov, T.A. Nagornova, V.S. Chernyshev, MATEC Web Conf. 23, 01051 (2015)

8. J. W. Cipolla Jr., H. Lang, S. K. Loyalka, J Chem Phys. 61, 69 (1974)

9. E. Ya. Gatapova, I.A. Graur, F. Sharipov, O.A. Kabov, Int. J. Heat Mass Transfer 83, $235(2015)$

10. E.Ya. Gatapova, R.A. Filipenko, Yu.V Lyulin, I.A. Graur, I.V. Marchuk, O. A. Kabov, Thermophysics and Aeromechanics 22, 701 (2015)

11. G.V. Kuznetsov, D.V. Feoktistov, E.G. Orlova, J. Eng. Phys. Thermophys. 89, 317 (2016)

12. D.V. Feoktistov, E.G. Orlova, A.G. Islamova, MATEC Web Conf. 23, 01054 (2015)

13. N.A. Yaryshev, Theoretical Fundamentals of Non-Stationary Temperature Measurements (Energoatomizdat, Leningrad, 1990) 